Relations industrielles

Industrial Relations

\title{
Workmen's Compensation - By Herman Miles Somers and Anne Ramsay Somers. New York: John Wiley \& Sons Inc., 1954. 344 pp.
}

\section{C.-Raymond Maranda}

Volume 13, numéro 2, avril 1958

URI : https://id.erudit.org/iderudit/1022453ar

DOI : https://doi.org/10.7202/1022453ar

Aller au sommaire du numéro

Éditeur(s)

Département des relations industrielles de l’Université Laval

ISSN

0034-379X (imprimé)

1703-8138 (numérique)

Découvrir la revue

Citer ce compte rendu

Maranda, C.-R. (1958). Compte rendu de [Workmen's Compensation - By Herman Miles Somers and Anne Ramsay Somers. New York: John Wiley \& Sons Inc., 1954. 344 pp.] Relations industrielles / Industrial Relations, 13(2), 237-238. https://doi.org/10.7202/1022453ar

Tous droits réservés (C Département des relations industrielles de l’Université Laval, 1958
Ce document est protégé par la loi sur le droit d'auteur. L'utilisation des services d'Érudit (y compris la reproduction) est assujettie à sa politique d'utilisation que vous pouvez consulter en ligne.

https://apropos.erudit.org/fr/usagers/politique-dutilisation/ 
tes sont soulevées se rapportant à la mobilité occupationnelle horizontale et verticale de cette catégorie de personnes. Mais c'est dans un autre ouvrage publié par les mêmes auteurs, Occupational Mobility in American Business and Industry, 1928-1952 (The University of Minnesota Press 1955) que l'on trouvera une présentation plus scientifique. Ici les auteurs ont voulu rendre abordable au grand public le fruit de leur recherche. Nous croyons qu'ils ont fait un excellent travail qui, tout en se lisant avec l'intérêt d'un roman, repose sur des données ne laissant pas place à la fiction et surtout répond à un besoin de connaître de plus près la personalité de ces gens qui contrôlent le pouvoir économique des Etats-Unis.

\section{G.D.}

Workmen's Compensation - By Herman Miles Somers and Anne Ramsay Somers. New York: John Wiley \& Sons Inc., 1954. 344 pp.

Cet ouvrage particulièrement utile à ceux qui s'intéressent aux questions d assurance applicables à la protection des travailleurs, est dominé par trois grandes idées: la prévention, l'assurabilité et la réhabilitation du handicapé professionnel.

Malgré les progrès accomplis dans le domaine de la prévention, il y eut, aux Etats-Unis, au delà de deux millions d'accidents en 1952. La gravité et la fréquence sont variables selon l'industrie et l'occupation.

Les auteurs expliquent que le problème causé par les accidents a son origine dans l'organisation impersonnelle de l'industrie, le travail à bon marché et la technologie moderne qui a créé une multitude d'opérations réalisées par des machines que l'individu ne peut ni comprendre, ni contrôler, et au rythme desquelles il doit s'adapter. De ces accidents, il résulte pour le travailleur, l'employeur et la société des pertes financières qu'il est impossible d'évaluer entièrement.

C'est seulement en 1907 qu'aux EtatsUnis, on a pris des mesures plus efficaces pour promouvoir la prévention des aocidents et offrir au travailleur une indemnité pour la perte de son salaire.
En 1920, on commença à s'occuper de la réhabilitation du travailleur accidenté. En deuxième partie, on explique les champs d'application de diverses lois en usage dans les Etats américains.

Au moyen d'une série de tableaux et de graphiques, on fait un exposé comparatif entre les Etats qui ont des plans d'assurance-accidents. Les informations obtenues de ces tableaux et graphiques nous renseignent sur l'application et l'étendue des différentes lois des accidents du travail, sur les bénéfices payés pour les incapacités totales permanentes ė temporaires; sur les périodes d'attente pour obtenir des bénéfices, sur les allocations et prestations payées aux veuves et aux orphelins, et sur les maladies professionnelles considérées au sens de la loi.

La dernière partie traite de réhabilitation. La prévention la mieux onganisée ne pourra jamais éliminer tous les accidents; les prestations, même les plus généreuses, ne pourront jamais remplacer ce qui est diminué, comme le prestige de l'individu et la compétence d'une main-d'oeuvre inutilisée.

Si nous ne pouvons éviter ni prévenir les tragédies causées par les accidents, nous devons, par ailleurs, disposer des moyens propres à redonner à l'individu toute la capacité physique possible, la compétence professionnelle nécessaire et la considération sociale qu'il est en droit d'espérer pour atteindre sa valeur d'homme.

Les auteurs puisent dans les volumes du Dr Henry Kessler et du Dr Howard Rush les données et principes essentiels du processus de réhabilitation.

Il définit ainsi le processus de la réhabilitation:

«The restoration of the handicapped to the fullest physical, mental, social, vocational and economic usefulness of which they are capable. »

Les auteurs citent quelques exemples d'individus réhabilités et montrent jusqu'à quel point la médecine a évolué dans ce sens.

On estime qu'il y a aux Etats-Unis entre 23 et 28 millions d'habitants souffrant d'une diminution physique ou mentale quelconque, dont deux millions environ auraient besoin d'être réhabilités avant d'être retournés au travail. 
Faut-il ajouter que les accidents de travail, de la rue et les infirmités congénitales fournissent annuellement, en moyenne, 250,000 handicapés de plus.

On est parfois enclin à croire que ce sont les guerres qui fournissent le contingent le plus considérable de personnes handicapées. Durant la dernière guerre, il y eut 20,500 amputés chez les militaires; durant cette période, les civils en comptaient 120,$000 ; 1,500$ des forces armées devinrent aveugles et pour les civils, 60,000 .

D'autres exemples sont donnés montrant l'évidence de ce problème. Les auteurs font une brève description du programme fédéral de réhabilitation, de celui des anciens combattants et des organismes des Etats américains sur qui repose, en définitive, la réalisation pratique de la réhabilitation.

Les auteurs, en terminant cette partie, soulignent l'aspect économique de la réhabilitation. Il est admis que pour chaque dollar dépensé par le Gouvernement fédéral à la réhabilitation, le travailleur handicapé remis dans le circuit économique paiera $\$ 10.00$, en taxes, au Gouvernement fédéral.

Ce volume contient de plus trois appendices dans lesquels les auteurs décrivent le système de compensation en Angleterre et en Ontario. Dans quelques lignes, ils résument trop brièvement le service de réhabilitation de la Commission des Accidents du Travail de l'Ontario, situé à Malton, lequel est, selon nos vues, le mieux organisé au Canada.

Les auteurs sont des économistes, et versés dans l'administration des assurances sociales. Dans ce volume, ils s'attardent à traiter de question d'assurabilité, de paiement de prestations aux accidentés, enfin à tout ce que comporte l'assurance-accidents.

Sans doute est-il question de réhabilitation. En fait, les auteurs consacrent une cinquantaine de pages à ce sujet; ils résument ce qui s'est fait aux EtatsUnis.

Nous croyons que ce volume peut intéresser plus particulièrement les spécialistes adonnés aux questions d'assuranice-accidents.

\section{C.-RAymond Maranda}

Making Management Human. By A.J. Marrow. New York: McGraw-Hill, 1957,241 pp.

M. Marrow est un psychologue, professeur au «New School for Social Research », et qui par surcroît est président de la «Harwood Manufacturing Corporation». Dans son volume, il rapporte les résultats qui furent atteints dans sa corporation depuis environ vingt ans, grâce à l'application de principes psychologigues aux différentes phases de l'administration du personmel. Marrow comprend parmi ses collaborateurs un chercheur comme Alex Bavelas. Dans de telles circonstances, les recherches entreprises sont toujours intéressantes, de même que les résultats.

Tout au long de son exposé, Marrow n'hésite pas à blâmer la gérance de l'entreprise si elle n'obtient pas de ses employés la coopération à laquelle elle s'attend. "Too few executives in industry today have an adequate conception of the values the average man seeks in work, or the different meanings work has for different people... The success of today's executive depends on cooperation in his organization, or his skill in getting things done by, for and through people ». Comme de nombreux exécutifs ignorent les besoins psychologiques de leurs subalternes, ils obtiennent le conformisme mais non la coopération, car ils ne saisissent pas le sens du mot motivation. Par suite de l'application grandissante des principes de l'automation, des situations déjà tendues vont certainement éclater. En effet, l'employé, délivré de ses préoccupations techniques, verra de plus en plus ses pensées accaparées par les problèmes psychologiques de sa position.

Par suite de leur comportement, certains patrons ne savent pas ce qui se passe réellement. Ordinairement, l'entourage rapporte au patron les informations qu'il s'attend à recevoir. Par son comportement agressif, autoritaire, et même antagoniste, il n'a jamais appris à écouter et encore moins à comprendre. Seul il connaît la solution. Il commencera à parler avant que son interlocuteur ait terminé son propre exposé. Dans les conférenoes ou les autres réunions. il prend la parole, il la conserve. Il exige et obtient un consentement unanime guand il propose un changement quelconque. Comme l'ont fait si bien 